\title{
Fabrication of various optical tissue phantoms by the spin-coating method
}

\author{
Jihoon Park \\ Myungjin $\mathrm{Ha}$ \\ Sungkon Yu \\ Byungjo Jung
}




\title{
Fabrication of various optical tissue phantoms by the spin-coating method
}

\author{
Jihoon Park, Myungjin Ha, Sungkon Yu, and Byungjo Jung* \\ Yonsei University, Department of Biomedical Engineering, 1 Yonseidae-gil, Wonju, Gangwon-do 220-710, Republic of Korea
}

\begin{abstract}
Although numerous studies have been performed to fabricate various optical tissue phantom (OTP) models, the fabrication of OTPs that simulate skin layers is laborious and time-consuming owing to the intricate characteristics of skin tissue. This study presents various OTP models that optically and structurally simulate the epidermis-dermis skin layer. The spin-coating method was employed to reproduce a uniform thin layer that mimics the epidermis layer, and the fabrication parameters were optimized for epoxy and silicone reference materials. Various OTP models simulating blood vessels and hyperpigmentation lesions were fabricated using the two reference materials to determine their feasibility. The suitability of each of the two reference materials for OTP fabrication was qualitatively evaluated by comparing the quality of the OTP models. ๑ 2016 Society of Photo-Optical Instrumentation Engineers (SPIE) [DOI: 10.1117/1.JBO.21.6.065008]
\end{abstract}

Keywords: optical tissue phantom; thin layer; spin-coating method; skin.

Paper 150850RR received Dec. 19, 2015; accepted for publication Jun. 13, 2016; published online Jun. 29, 2016.

\section{Introduction}

Various optical tissue phantoms (OTPs) that simulate the geometric structures and optical properties of biological tissues have been developed and widely used for therapeutic and diagnostic purposes in biomedical optics over the past two decades. ${ }^{1-7}$ Human skin tissue is mainly divided into three layers - the epidermis, dermis, and subcutaneous layer-each with different optical properties and thicknesses. ${ }^{8}$ Although various OTP fabrication methods have been studied, ${ }^{9-12}$ an OTP that mimics skin layers has not yet been achieved owing to the intricate optical properties of skin, which are dependent upon the wavelength and the structural inhomogeneity.

To fabricate an OTP that mimics skin tissue, the following must be considered: ${ }^{13}$ (1) the optical properties of dominant chromospheres such as hemoglobin and melanin, ${ }^{14}(2)$ the scattering caused by the different refractive indices of tissue components, ${ }^{15}$ and (3) the geometry of blood vessels ${ }^{16}$ and pigmented skin lesions. ${ }^{7,17}$ In this study, Indian ink and titanium dioxide $\left(\mathrm{TiO}_{2}\right)$, which are commonly used in OTP studies, ${ }^{18-21}$ were chosen as absorber and scatterer, respectively. Absorption and reduced scattering coefficients were derived as a function of the concentrations of these materials to fabricate an OTP mimicking the optical properties of skin at $785 \mathrm{~nm}$.

The epidermis, in particular, is considered the most difficult layer to fabricate because of its microscale thickness. Despite this, various methods ${ }^{22-25}$ have been proposed to fabricate a thin epidermis layer. A previous study demonstrated the feasibility of the spin-coating method (SCM) in the fabrication of a thin-layer OTP with epoxy, ${ }^{26}$ and other studies ${ }^{6,27}$ have introduced silicone OTPs consisting of thin films with better quality. In this study, the SCM was used to mimic the epidermis layer with both epoxy and silicone and their outcomes were compared.
To investigate the reliability and objectivity of the resulting OTP, the optical properties of each OTP layer were assessed with a double-integrating sphere system and inverse addingdoubling (IAD) program. In addition, the thickness of each layer was measured with a digital Vernier caliper and crosssectional images. Epidermis-dermis structure-based OTPs simulating blood vessels and pigmented skin disorders were fabricated to demonstrate their practical effectiveness. Although their optical properties were only assessed at a single wavelength, we present feasible OTPs that can be used in optical diagnostics and therapies, which are based on a single wavelength, such as laser-based techniques.

\section{Materials and Methods}

\subsection{Preparation for Optical Tissue Phantom Fabrication}

\subsubsection{Epidermis-dermis layer}

The epidermis and dermis layers limit light propagation in skin tissue. Light may be propagated only a few millimeters in skin tissue because of the light scattering and absorption properties in both of these skin layers. For this reason, the subcutaneous fat layer was not considered in this study, although, it plays an important role physiologically. An OTP of the epidermis-dermis layer was designed by considering both the optical properties and thickness as detailed in previous studies ${ }^{28-31}$ as shown in Table 1.

\subsubsection{Material selection}

Black Indian ink (951 Black Indian ink, Winsor \& Newton, France) and the anatase form of $\mathrm{TiO}_{2}$ (T0407, Samchun Chemical, Korea) were chosen to mimic the absorption 
Table 1 Predetermined characteristics of real tissues for fabrication of OTPs.

\begin{tabular}{|c|c|c|c|c|}
\hline \multirow[b]{2}{*}{$\begin{array}{l}\text { Tissue } \\
\text { components }\end{array}$} & \multicolumn{2}{|c|}{ Optical property at $785 \mathrm{~nm}$} & \multirow[b]{2}{*}{$\begin{array}{l}\text { Thickness } \\
(\mathrm{mm})\end{array}$} & \multirow[b]{2}{*}{ Remarks } \\
\hline & $\begin{array}{c}\text { Absorption } \\
\text { coefficient } \\
(1 / \mathrm{mm})\end{array}$ & $\begin{array}{c}\text { Reduced } \\
\text { scattering } \\
\text { coefficient } \\
(1 / \mathrm{mm})\end{array}$ & & \\
\hline Epidermis & 0.18 & 3.70 & 0.10 & $\begin{array}{l}\text { Fabricated } \\
\text { by SCM }\end{array}$ \\
\hline Dermis & 0.12 & 2.30 & 2.00 & $\begin{array}{l}\text { Fabricated } \\
\text { by petri dish } \\
\text { molding }\end{array}$ \\
\hline $\begin{array}{l}\text { Blood } \\
\text { vessel }\end{array}$ & - & - & - & $\begin{array}{l}\text { Silicone tube } \\
\text { located at } \\
\text { 2-mm depth }\end{array}$ \\
\hline Blood & - & - & - & Porcine blood \\
\hline $\begin{array}{l}\text { Pigment } \\
\text { (1\% melanin) }\end{array}$ & 1.6 & - & 10.00 & $\begin{array}{l}\text { Indian ink } \\
\text { and epoxy }\end{array}$ \\
\hline
\end{tabular}

coefficient and reduced scattering coefficient, respectively, of each skin layer at $785 \mathrm{~nm}$. Sample OTPs were prepared with five different concentrations of Indian ink and $\mathrm{TiO}_{2}$ for optical property measurement: $200,400,600,800$, and $1000 \mathrm{mg}$ of Indian ink and 100, 200, 300, 400, and $500 \mathrm{mg}$ of $\mathrm{TiO}_{2}$ in $100 \mathrm{~mL}$ of epoxy or silicone. Next, the correlation between the optical properties of the sample OTPs and the concentrations of the materials was derived.

A transparent silicone tube (T05*1.0, HSW, Korea) with an inner diameter of $0.5 \mathrm{~mm}$ and an outer diameter of $1 \mathrm{~mm}$ was used to mimic blood vessel structures in the skin tissue. The optical properties of the tube were not assessed, however, because the optical properties of the dermis layer include those of the dermal components, including blood vessels.

Pigmentation OTP model was prepared to be $2.5 \mathrm{~cm}$ in diameter and $1 \mathrm{~cm}$ in thickness to mimic a hyperpigmented melanin lesion by mixing epoxy and Indian ink. The absorption coefficient of the melanin lesion was determined to be $1.6 \mathrm{~mm}^{-1}$, which is the same as that of a $1 \%$ concentration of melanin. ${ }^{7}$

\subsubsection{Spin-coating method}

A spin coater (ACE-200, Dong Ah Trade Corp., Korea) was used to produce a thin, $100-\mu \mathrm{m}$ layer. The coating thickness produced by the SCM was investigated as a function of spin speed for both epoxy and silicone reference materials. In this experiment, the acceleration time was $5 \mathrm{~s}$ and the coating duration was $60 \mathrm{~s}$ for both reference materials. The epoxy mixture was hardened at room temperature for $4 \mathrm{~h}$ to increase the viscosity of spin coating, while the silicone mixture was coated immediately after being prepared.

Each reference material was coated three times at 1400 , 1800, 2200, 2600, and $3000 \mathrm{rpm}$, and then five points on each sample were randomly selected for thickness measurement, with cross-sectional images captured by a digital microscope (KH-7700, HIROX, Japan).

\subsection{Fabrication of Optical Tissue Phantoms}

\subsubsection{Preparation of optical tissue phantom mixture}

Figure 1 shows the procedure for the fabrication of the OTP for the epidermis-dermis layer. For the epoxy OTP, Indian ink and $\mathrm{TiO}_{2}$ were added to pure epoxy (KE-300NS, KPS, Korea) and then homogenized for $5 \mathrm{~min}$. The mixture, heated by ultrasound energy, was cooled down to room temperature to ensure optical and chemical stability and was then subsequently mixed with a hardener (KH-700NS) in a 3:1 volume ratio.

The silicone OTP was prepared using the same procedure except that the Indian ink and $\mathrm{TiO}_{2}$ were added to the hardener (CAT-RG, SinEtsu, Japan) first, rather than adding them to the pure silicone (KE-1606). This is because silicone is easily damaged by ultrasound energy during homogenization. The silicone and hardener were mixed in a 9:1 volume ratio.

\subsubsection{Double-layer optical tissue phantom}

The dermis OTP mixture was poured into a Petri dish, and then degassing was performed at room temperature until all gasses were removed. A vacuum chamber was used to degas the silicone OTP mixture, which had relatively more air bubbles. After curing in an incubator for $3 \mathrm{~h}$ at $55^{\circ} \mathrm{C}$, the bottom of the dermis OTP was used for epidermal spin coating.

\subsubsection{Blood vessel optical tissue phantom}

The epidermis-dermis OTP included a silicone tube array to simulate blood vessel structure in skin layers and blood circulation. The tubing was placed $2 \mathrm{~mm}$ above the bottom of the petri dish mold and then sealed with glue. Silicone, which has a low viscosity in the liquid state and high elasticity in the cured state, was used to complement the material properties of the silicone tube. The OTP mixture for the dermis was poured into the mold up to a height of $2 \mathrm{~mm}$. After degassing, the OTP was cured in an incubator for $3 \mathrm{~h}$ at $55^{\circ} \mathrm{C}$. The epidermal OTP mixture was spin coated to the flat bottom of the cured dermal blood vessel OTP.

\subsubsection{Pigmentation optical tissue phantom}

OTP mimicking pigmentation skin lesions was fabricated using epoxy as a reference material, which minimizes air bubbles created during fabrication of the thick sample. Thin wires were used to fix three cylindrical rod pigmentation models at heights of 1,2 , and $3 \mathrm{~mm}$ from the bottom of the Petri dish to simulate pigmentation skin lesions at different depths. Dermal OTP mixture was poured over these (up to a height of $\sim 1 \mathrm{~cm}$ ) to fix the three rod pigmentation models and then was cured for $6 \mathrm{~h}$ at $55^{\circ}$ C. Finally, the thin wires were removed, and spin coating was used to add the epidermal OTP layer.

\subsection{Characterization of Optical Tissue Phantoms}

\subsubsection{Measurement of optical properties}

A double-integrating sphere system and Prahl's IAD method ${ }^{32,33}$ were used to assess the optical properties of the OTPs and to compare them with the predetermined values at a wavelength of $785 \mathrm{~nm}$, as shown in Fig. 2. A broadband halogen lamp (HL-2000, Ocean Optics) was used as a light source and guided to one of the double-integrating spheres (AvaSphere30, Avantes, Netherlands), which had an inner diameter of 
Stage 1. Preparation of phantom suspension
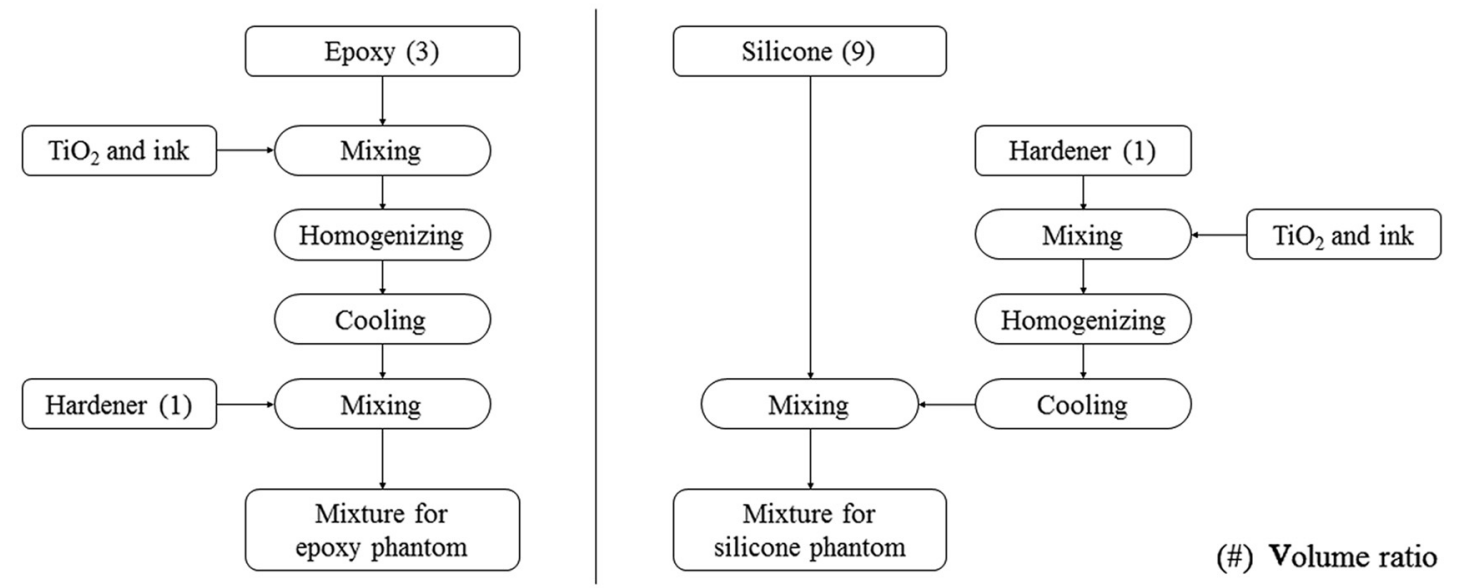

Stage 2. Phantom fabrication

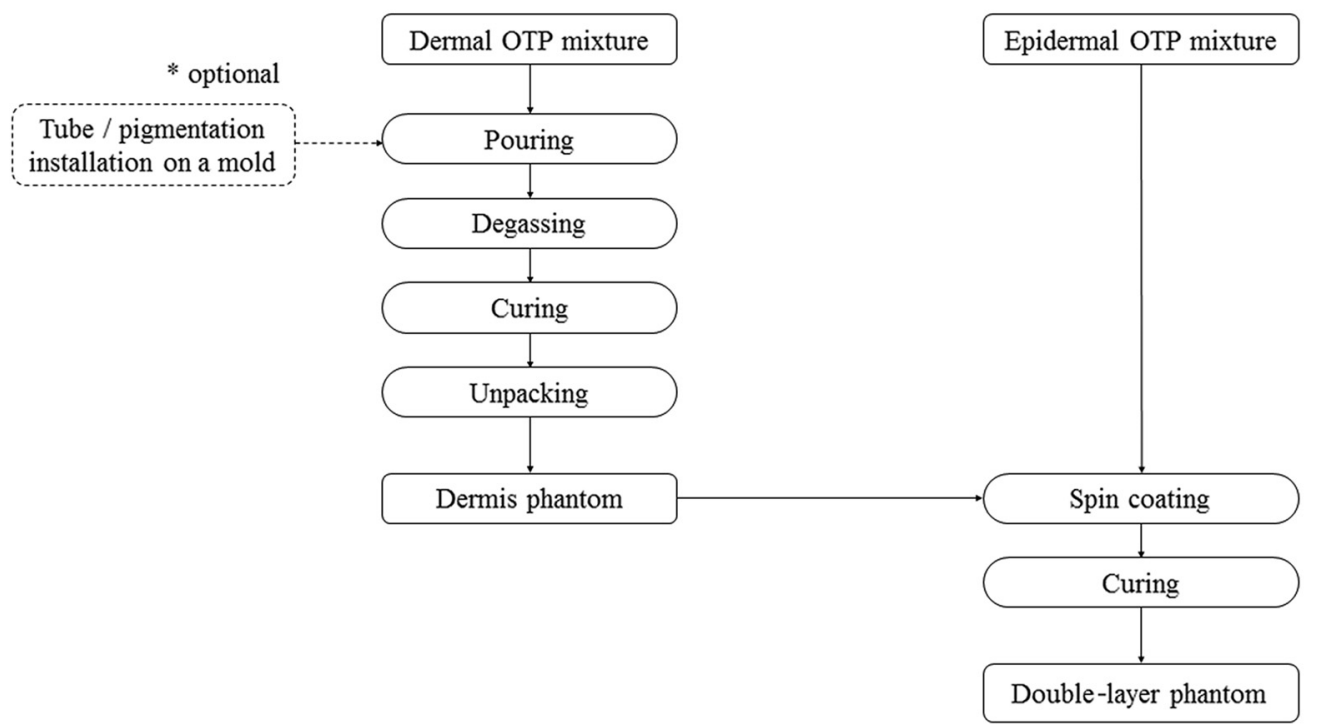

Fig. 1 Procedure for fabrication of double-layer OTP.

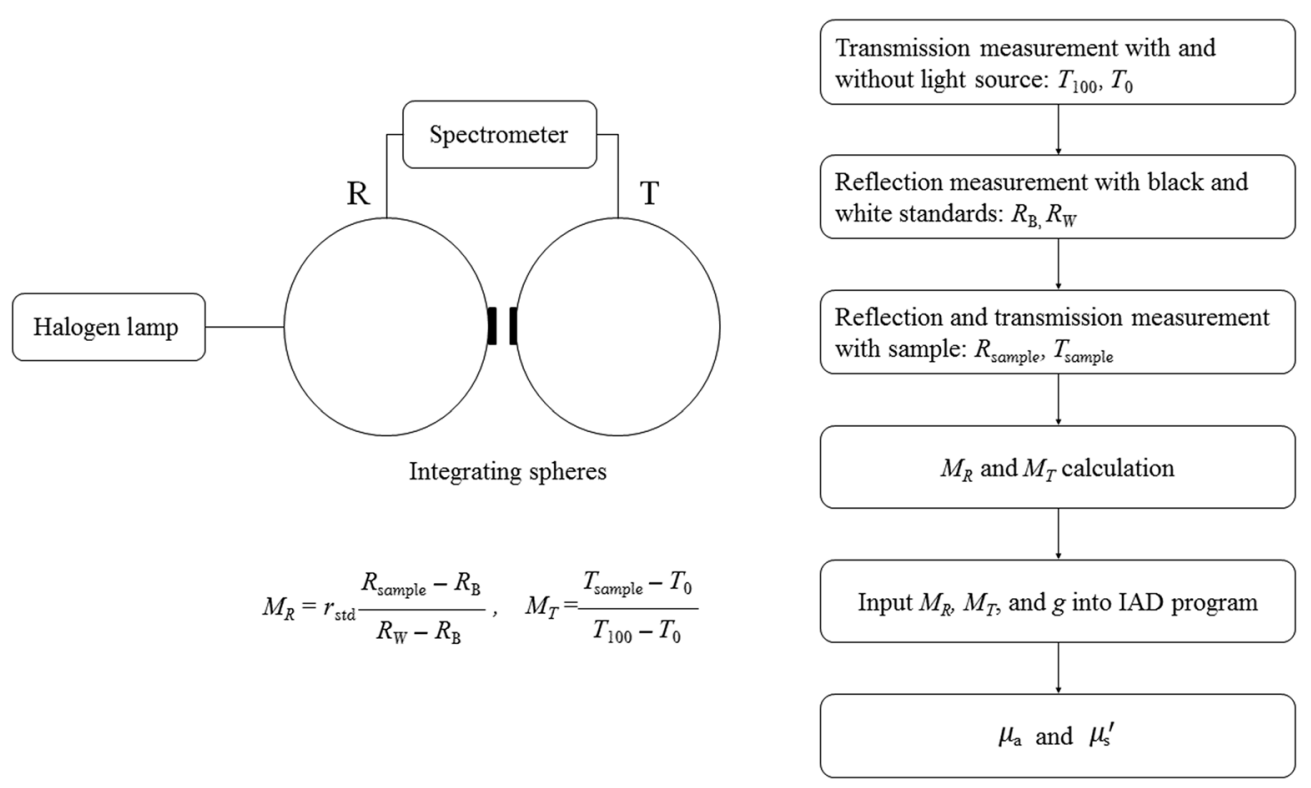

Fig. 2 Double-integrating sphere system and flowchart for derivation of absorption and reduced scattering coefficients from IAD program. 
$30 \mathrm{~mm}$, a 6-mm sample port, and a baffle between ports. The measurement ports of the two integrating spheres were guided to a spectrometer (USB4000, Ocean Optics) through an optical fiber (FC-IR400-2, Avantes, Netherlands) with a diameter of $400 \mu \mathrm{m}$. Three samples were prepared for each measurement, and five measurement points were randomly selected per sample. Total reflectance $\left(M_{R}\right)$ and transmittance $\left(M_{T}\right)$ were calculated based on measured values and used in the IAD program along with the anisotropy factor ( $g$ value), which was experimentally determined. ${ }^{34}$

\subsubsection{Thickness measurements}

Cross-sectional images of the epidermis-dermis OTP were obtained using digital microscopic and optical coherence tomographic (OCT; OCP930SR, Thorlabs) imaging modalities. The thickness of the dermis layer was determined with a digital Vernier caliper and that of the epidermis layer was determined with the cross-sectional image obtained from the digital microscope. Five measurement points on the OTP were randomly selected, and average values were calculated.

\subsubsection{Laser speckle contrast imaging for blood vessel optical tissue phantom}

The surface image of the blood vessel OTP was captured with a near-infrared (NIR) camera (Manta G-145B, Allied Vision Technologies GmbH, Germany). The laser speckle contrast imaging modality, which is a powerful tool for full-field imaging of blood flow, ${ }^{35}$ was used to demonstrate the functional performance of the blood vessel OTP. The ends of the silicone tube were connected to a peristaltic pump (PP-150D, Poong Lim, Korea) and then porcine blood, which was obtained at an abattoir and archived in heparin-coated tubes (BD Vacutainer, BD), was circulated at $60 \mathrm{rpm}$ to simulate blood flow in the blood vessel. Thirty laser speckle images were captured with a 785-nm diode laser (L785P090, Thorlabs), and then the laser speckle contrast index of each image was calculated and averaged using MATLAB, as previously described. ${ }^{36}$ The scale of the index ranged from 0.27 to 0.47 of the maximum laser speckle contrast.

\subsubsection{Intensity profile of pigmentation optical tissue phantom}

A surface image of the pigmentation OTP was obtained with a cross-polarization system (MAG Vision-2000, Optobiomed, Korea), consisting of polarizers, ring light LEDs, and a digital single-lens reflex camera (500D, Canon, Japan), in order to identify contrast differences between skin pigmentation lesions at different depths. The image intensity profile was obtained along the center of the OTP image and was plotted in grayscale.

\section{Results}

\subsection{Characteristics of Materials}

As shown in Fig. 3, the absorption coefficient $\left(\mu_{\mathrm{a}}\right)$ of Indian ink and reduced scattering coefficient $\left(\mu_{\mathrm{s}}^{\prime}\right)$ of $\mathrm{TiO}_{2}$ were calculated as a function of the concentration of the reference materials from a linear equation derived by regression analysis as follows:

$\mu_{a_{-} \text {epoxy }}=0.073 c_{\mathrm{ink}}$,
$\mu_{\text {s_epoxy }}^{\prime}=0.628 c_{\mathrm{TiO}_{2}}+0.266$,

and

$\mu_{a_{\text {_silicone }}}=0.087 c_{\mathrm{ink}}$,

$\mu_{\text {s_epoxy }}^{\prime}=0.852 c_{\mathrm{TiO}_{2}}+0.184$

where $c_{\text {ink }}$ and $c_{\mathrm{TiO}_{2}}$ represent the weight of the ink and $\mathrm{TiO}_{2}$ in $1 \mathrm{~mL}$ of reference materials. Using the above equations, the concentrations of Indian ink and $\mathrm{TiO}_{2}$ in each OTP layer were determined, as shown in Table 2.

\subsection{Coating Thickness as a Function of Material and Spin-Coating Speed}

We observed some variation in coating thickness as a function of spin-coating speed for the epoxy and silicone reference materials (Fig. 4). Silicone was more suitable for SCM in terms of coating uniformity. Figure 5 shows the surface and cross-sectional OCT images of single-layer epoxy and silicone OTPs coated on the surface of the Petri dish. While the silicone OTP exhibited a flat and uniform coating layer, the epoxy OTP contained some holes and cracks in the coat. For creation of the $100-\mu \mathrm{m}$ epidermal layer, spin speeds of 3000 and $2200 \mathrm{rpm}$ were optimal for epoxy and silicone, respectively (Table 2).

\subsection{Optical Properties of Optical Tissue Phantom}

Measurements of the optical properties of epoxy and silicone OTPs (Table 2) demonstrated that measured absorption and reduced scattering coefficients were close to the expected values (within 10\%). This indicates that the OTP mixture was fully homogenized and exhibited precise concentrations of materials.
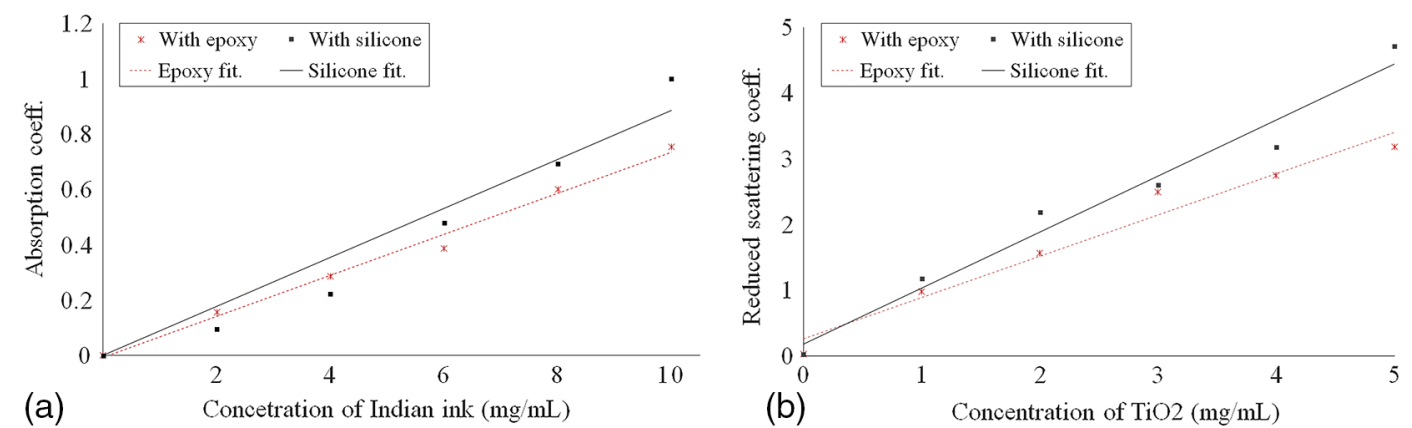

Fig. 3 Absorption and reduced scattering coefficients as a function of Indian ink and $\mathrm{TiO}_{2}$ concentration. 
Table 2 Measured optical properties and thicknesses of OTPs.

\begin{tabular}{|c|c|c|c|c|}
\hline \multirow{2}{*}{\multicolumn{2}{|c|}{$\begin{array}{l}\text { Reference materials of tissue } \\
\text { layer }\end{array}$}} & \multicolumn{2}{|c|}{ Measured optical property at $785 \mathrm{~nm}$} & \multirow[b]{2}{*}{$\begin{array}{l}\text { Thickness (mm) } \\
\text { (Spin speed) }\end{array}$} \\
\hline & & $\begin{array}{c}\text { Absorption } \\
\text { coefficient }\left(\mathrm{mm}^{-1}\right)\left(c_{\text {ink }}\right)\end{array}$ & $\begin{array}{l}\text { Reduced scattering } \\
\text { coefficient }\left(\mathrm{mm}^{-1}\right)\left(c_{\mathrm{TiO}_{2}}\right)\end{array}$ & \\
\hline \multirow[t]{2}{*}{ Epoxy } & Epidermis & $0.19( \pm 0.03)(2.5 \mathrm{mg} / \mathrm{mL})$ & $3.87( \pm 0.29)(5.5 \mathrm{mg} / \mathrm{mL})$ & $0.10(2800 \mathrm{rpm})$ \\
\hline & Dermis & $0.14( \pm 0.03)(1.6 \mathrm{mg} / \mathrm{mL})$ & $2.38( \pm 0.18)(3.2 \mathrm{mg} / \mathrm{mL})$ & 2.00 \\
\hline \multirow[t]{2}{*}{ Silicone } & Epidermis & $0.20( \pm 0.01)(2.1 \mathrm{mg} / \mathrm{mL})$ & $3.63( \pm 0.27)(4.1 \mathrm{mg} / \mathrm{mL})$ & $0.91(2200 \mathrm{rpm})$ \\
\hline & Dermis & $0.11( \pm 0.03)(1.4 \mathrm{mg} / \mathrm{mL})$ & $2.50( \pm 0.25)(2.5 \mathrm{mg} / \mathrm{mL})$ & 2.00 \\
\hline
\end{tabular}

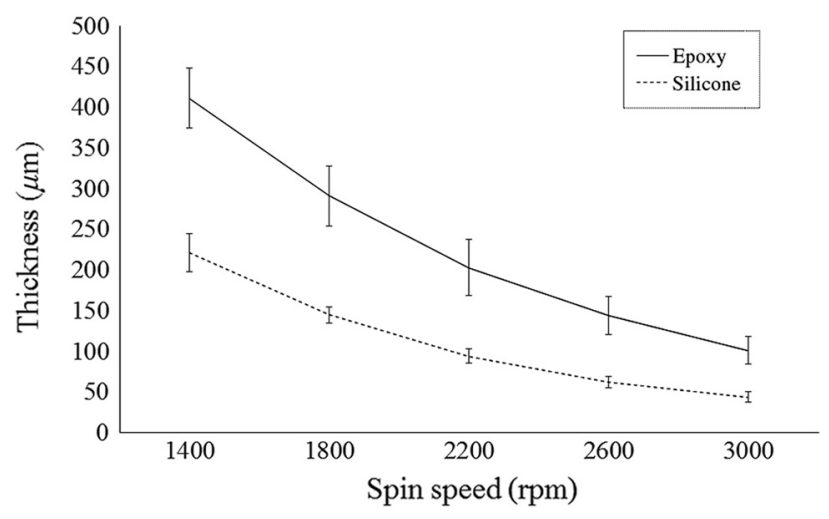

Fig. 4 Variation in coating thickness due to materials and spin coating speeds.

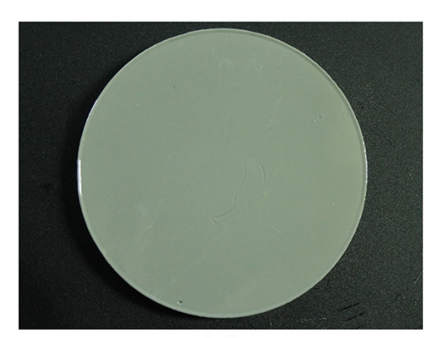

(a)

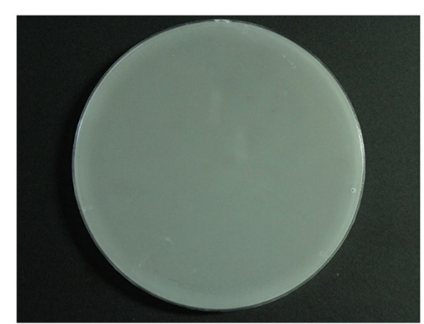

(c)

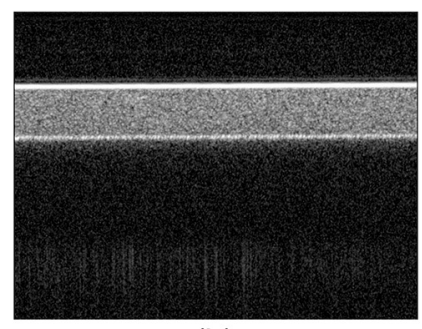

(b)

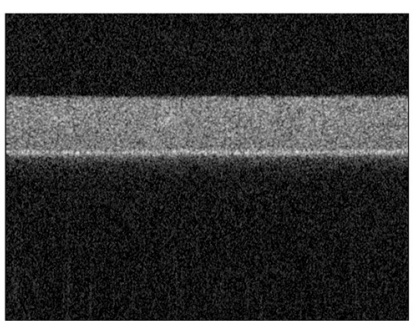

(d)
Fig. 5 Spin-coated thin-layer OTPs: (a) surface image and (b) OCT image of epoxy OTP; (c) surface image and (d) OCT image of silicone OTP.

\subsection{Cross-Sectional Images of Optical Tissue Phantom}

Cross-sectional images of OTPs acquired using the OCT (Fig. 5), and the digital microscope (Fig. 6) show that there are some differences between epoxy and silicone OTPs, such as differences in color and scattering signal in the OCT images.
This may be because optical properties were only assessed at $785 \mathrm{~nm}$. In terms of thickness, however, both OCT and microscope images show that the measured thicknesses are similar to the desired values, with uniform coating at the microscale. Although the epoxy OTP exhibited less uniformity than silicone with some pores and cracks, cross-sectional images show mostly flat and uniform coating layers for both reference materials.

The average epidermal thicknesses of epoxy and silicone OTPs were $0.108( \pm 0.018) \mathrm{mm}$ and $0.097( \pm 0.009) \mathrm{mm}$, respectively, and the average dermal thicknesses were 2.10 $( \pm 0.09) \mathrm{mm}$ and $1.89( \pm 0.17) \mathrm{mm}$, respectively.

\subsection{Assessment of Optical Tissue Phantom Models}

The laser speckle contrast image (LSCI) showed that blood flow in the silicone tube exhibited a higher speckle contrast index than the surrounding epidermal-dermal OTP, as shown in Fig. 7. Although the LSCI reflected a laser speckle contrast index of only $60 \mathrm{rpm}$, we confirmed that the total level of the speckle index was proportional to the velocity of the flow.

We captured a cross-polarization image of the pigmentation OTP and determined the intensity profile along the center of the image (Fig. 8). The cross-polarization image demonstrated that the deeper the pigmentation model was located, the more shadow was blurred. The intensity profile indicates that deeper pigmentation models exhibited lower intensities and, therefore, higher scattering.

\section{Discussion}

Fabrication of OTPs mimicking the thickness and optical properties of the skin has been previously regarded as complicated and time-consuming. This study endeavored to fabricate a thinlayer OTP using SCM to simulate an epidermis-dermis skin layer, which is difficult to mimic. We presented not only this thin-layer OTP but also various other OTP models and optimized the fabrication parameters for two reference materials, silicone and epoxy.

Although an OTP consisting of Indian ink and $\mathrm{TiO}_{2}$ cannot simulate the optical spectrum of skin tissue, mimicking the optical properties of skin at a single wavelength can be useful for laser-based applications, such as laser speckle imaging and laser propagation in skin. Future studies using laser speckle image analysis and vein detection techniques to analyze a blood vessel OTP will be performed to verify the effectiveness of the OTPs presented here.

Although silicone, with its relatively high viscosity, was more suitable for SCM, epoxy was also found to be feasible 


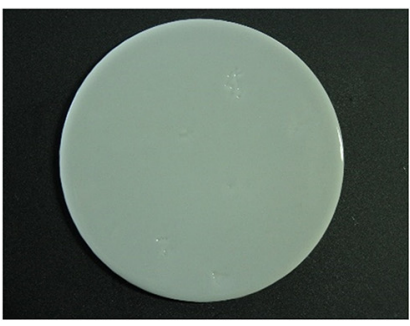

(a)

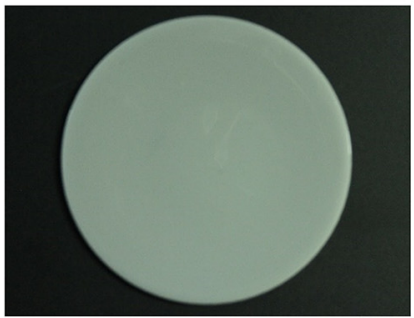

(d)

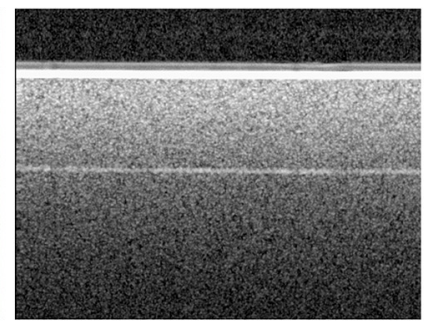

(b)

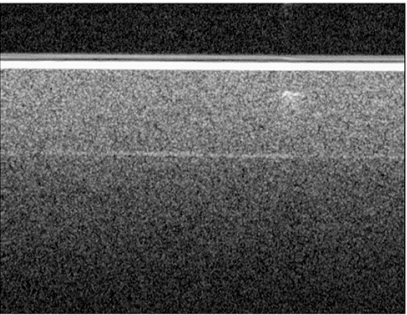

(e)

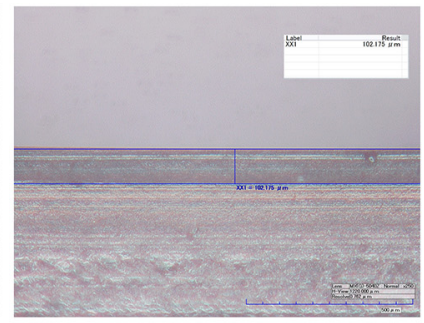

(c)

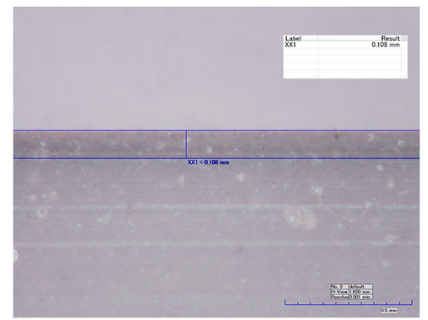

(f)

Fig. 6 Double-layer OTPs mimicking the epidermis-dermis structure: (a) surface image, (b) OCT image, and (c) digital microscope image of epoxy OTP; (d) surface image, (e) OCT image, and (f) digital microscope image of silicone OTP.

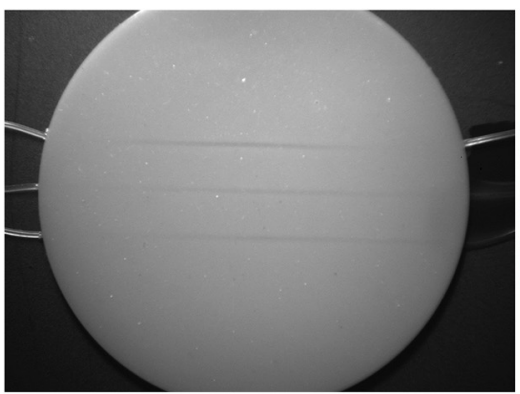

(a)

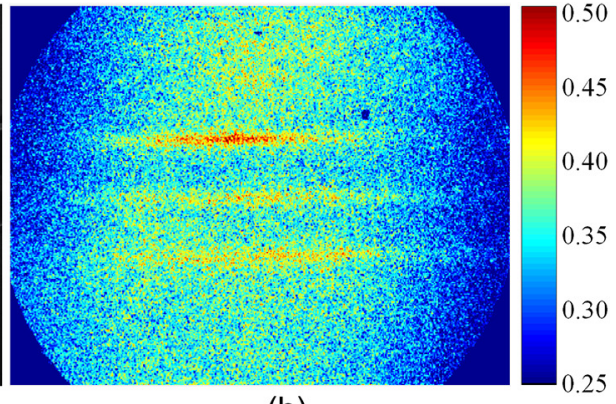

(b)

Fig. 7 Blood vessel OTP: (a) NIR image captured by an NIR camera under NIR light (uniform ring light LED) and (b) LSCl, in which porcine blood was circulated through a silicone tube with a peristaltic pump.

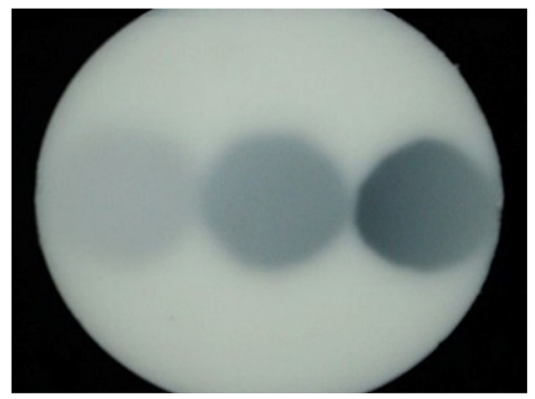

(a)

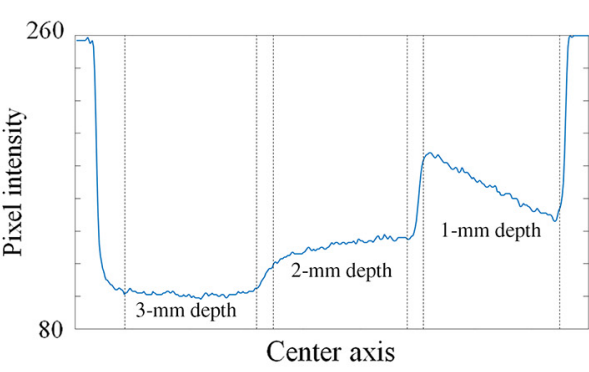

(b)

Fig. 8 Pigmentation OTP: (a) cross-polarization image and (b) intensity profile along the center of the cross-polarization image showing the relative depth of the pigmentation OTP, in which greater pixel intensity indicates a more shallow depth.

for OTP fabrication and presents the additional benefits of a simple manufacturing procedure, low cost, and frequent use in other OTP studies. In attempting to improve the coating quality of an epoxy with relatively low viscosity, we found that the viscosity of the epoxy solution tends to increase during the hardening process. Although we attempted to control the viscosity with hardening time to improve the spin coating results, the cross-polarization surface images of OTPs show that the coating quality of silicone was still higher than that of epoxy. Excessive increases in the viscosity and hardening of the medium may obstruct the natural degassing process and ultimately generate holes and cracks in the surface of the OTP. The OTP fabrication process is simpler with silicone because of its high viscosity at room temperature. However, its stickiness and high viscosity in 
the liquid state obstruct homogenization and make handling and quantification difficult. In contrast, epoxy is easier to handle and quantify because of its low viscosity in the liquid state and low mixture ratio, and, unlike silicone, it is rarely necessary to use a vacuum pump for degassing.

The spin coating parameters used in this experiment, such as acceleration time and coating duration for each coating material, were empirically predetermined. We found that the effect of increasing the viscosity of the epoxy on the coating quality was dependent on the acceleration time, as not using the appropriate acceleration time for the reference materials commonly causes spin coating defects. Though we did not find a correlation between acceleration time and coating quality, a coating duration of more than $40 \mathrm{~s}$ was generally required to spread the material sufficiently and uniformly.

In this study, silicone and epoxy were used to fabricate OTP models mimicking blood vessels and pigmentation skin lesions, respectively. Silicone was chosen for use in the blood vessel OTP because of the similarities between the elasticity and optical characteristics of the silicone tube. LSCI was used to assess the performance of the blood vessel OTP, and the range of the laser speckle contrast index was configured at 0.27 to 0.47 to maximize the laser speckle contrast, although the original LSCI exhibited enough image contrast. We confirmed that, as expected, the laser speckle contrast was higher around the silicone tube than the surrounding region. In addition, the total laser speckle contrast index was proportional to the speed of the blood flow, demonstrating the ability of the blood vessel OTP to simulate simple blood circulation.

For the pigmentation OTP, epoxy was used as a reference material due to its low viscosity as a liquid and stiffness as a solid. Such characteristics were useful in making the three small OTP pigmentation models and in removing the air bubbles between the pigmentation models and the dermal OTP mixture. Although the pigmentation OTP was thicker $(\sim 1 \mathrm{~cm})$ than the other OTPs, this was necessary for fixation of the pigmentation models after removal of the thin wires. Cross-polarization imaging was used to obtain a clear image contrast between the three pigmentation models and the surrounding region, followed by black-white reversal in grayscale to visualize the result. The relative depth of the pigmentation model can be inferred from its image contrast of pigmentation OTP. Analysis of cross-polarization images and grayscale intensity profile may, therefore, be useful in evaluating the depth and geometry of pigmentation OTPs.

Given our findings on the relative advantages and disadvantages of the epoxy and silicone reference materials in simulating human skin tissue using SCM, we conclude that in some cases, silicone may be the more suitable material for OTP fabrication, while in other cases, epoxy may be more appropriate. Our results provide a foundation for further study of the fabrication of OTPs for biomedical optics applications.

\section{Acknowledgments}

This research was financially supported by the Ministry of Trade, Industry and Energy and Korea Institute for Advancement of Technology.

\section{References}

1. B. W. Pogue and M. S. Patterson, "Review of tissue simulating phantoms for optical spectroscopy, imaging and dosimetry," J. Biomed. Opt. 11(4), 041102 (2006).
2. J. Bouchard et al., "Reference optical phantoms for diffuse optical spectroscopy. Part 1-error analysis of a time resolved transmittance characterization method," Opt. Express 18(11), 11495-11507 (2010).

3. B. Leh et al., "Optical phantoms with variable properties and geometries for diffuse and fluorescence optical spectroscopy," J. Biomed. Opt. 17(10), 108001 (2012).

4. D. Wang, Y. Chen, and J. T. C. Liu, "A liquid optical phantom with tissue-like heterogeneities for confocal microscopy," Biomed. Opt. Express 3(12), 3153-3160 (2012).

5. M. N. Iizuka, M. D. Sherar, and I. A. Vitkin, "Optical phantom materials for near infrared laser photocoagulation studies," Lasers Surg. Med. 25(2), 159-169 (1999).

6. J. Baxi et al., "Retina-simulating phantom for optical coherence tomography," J. Biomed. Opt. 19(2), 021106 (2014).

7. M. Lualdi et al., "Development of simulated pigmented lesions in an optical skin-tissue phantom: experimental measurements in the visible and near infrared," J. Laser Appl. 14(2), 122 (2002).

8. A. N. Bashkatov et al., "Optical properties of human skin, subcutaneous and mucous tissues in the wavelength range from 400 to $2000 \mathrm{~nm}$," J. Phys. D Appl. Phys. 38(15), 2543-2555 (2005).

9. R. B. Saager et al., "Multilayer silicone phantoms for the evaluation of quantitative optical techniques in skin imaging," Proc. SPIE 7567, 756706 (2010).

10. S. Tseng et al., "Determination of optical properties of superficial volumes of layered tissue phantoms," IEEE Trans. Biomed. Eng. 55(1), 335-339 (2008).

11. J. M. Schmitt, G. X. Zhou, and E. C. Walker, "Multilayer model of photon diffusion in skin," J. Opt. Soc. Am. A 7(11), 2141-2153 (1990).

12. P. Urso et al., "Skin and cutaneous melanocytic lesion simulation in biomedical optics with multilayered phantoms," Phys. Med. Biol. 52(10), N229-N239 (2007).

13. T. Lister, P. A. Wright, and P. H. Chappell, "Optical properties of human skin,” J. Biomed. Opt. 17(9), 090901 (2012).

14. I. Nishidate, Y. Aizu, and H. Mishina, "Estimation of melanin and hemoglobin in skin tissue using multiple regression analysis aided by Monte Carlo simulation," J. Biomed. Opt. 9(4), 700-710 (2004).

15. S. Nickell et al., "Anisotropy of light propagation in human skin," Phys. Med. Biol. 45(10), 2873-2886 (2000).

16. I. Nishidate, Y. Aizul, and H. Mishinal, "Estimation of bluish appearance of veins in skin tissue using spectrocolorimetry," Opt. Rev. 9(6), 269-276 (2002).

17. A. Mazzoli, R. Munaretto, and L. Scalise, "Preliminary results on the use of a noninvasive instrument for the evaluation of the depth of pigmented skin lesions: numerical simulations and experimental measurements," Lasers Med. Sci. 25(3), 403-410 (2010).

18. P. D. Ninni, F Martelli, and G. Zaccanti, "The use of India ink in tissuesimulating phantoms," Opt. Express 18(26), 26854-26865 (2010).

19. V. V. Tuchin et al., "Finger tissue model and blood perfused skin tissue phantom," Proc. SPIE 7898, $78980 \mathrm{Z}$ (2011).

20. I. V. Krasnikov, A. Y. Seteikin, and A. P. Popov, "Simulation of the effect of photoprotective titanium dioxide $\left(\mathrm{TiO}_{2}\right)$ and zinc oxide $(\mathrm{ZnO})$ nanoparticles on the thermal response and optical characteristics of skin," Opt. Spectrosc. 118(4), 668-673 (2015).

21. F. Ayers et al., "Fabrication and characterization of silicone-based tissue phantoms with tunable optical properties in the visible and near infrared domain," Proc. SPIE 6870, 687007 (2008).

22. T. Bergmanna et al., "Development of a skin phantom of the epidermis and evaluation by using fluorescence techniques," Proc. SPIE 7906, 79060T (2011).

23. R. C. Chang et al., "Fabrication and characterization of a multilayered optical tissue model with embedded scattering microspheres in polymeric materials," Biomed. Opt. Express 3(6), 1326-1339 (2012).

24. S. H. Tseng, A. Grant, and A. J. Durkin, "In vivo determination of skin near-infrared optical properties using diffuse optical spectroscopy," J. Biomed. Opt. 13(1), 014016 (2008).

25. T. L. Troy and S. N. Thennadil, "Optical properties of human skin in the near infrared wavelength range of 1000 to $2200 \mathrm{~nm}$," J. Biomed. Opt. 6(2), 167-176 (2001).

26. Y. Bae et al., "Fabrication of a thin-layer solid optical tissue phantom by a spin-coating method: pilot study," J. Biomed. Opt. 18(2), 025006 (2013). 
27. A. Agrawal et al., "Multilayer thin-film phantoms for axial contrast transfer function measurement in optical coherence tomography," Biomed. Opt. Express 4(7), 1166-1175 (2013).

28. E. Salomatina et al., "Optical properties of normal and cancerous human skin in the visible and near-infrared spectral range," J. Biomed. Opt. 11(6), 064026 (2006).

29. C. R. Simpson et al., "Near-infrared optical properties of ex vivo human skin and subcutaneous tissues measured using the Monte Carlo inversion technique," Phys. Med. Biol. 43(9), 2465 (1998).

30. J. Sandby-Moller, T. Poulsen, and H. C. Wulf, "Epidermal thickness at different body sites: relationship to age, gender, pigmentation, blood content, skin type and smoking habits," Acta Derm.-Venereol. 83(6), 410-413 (2003).

31. T. Gambichler et al., "In vivo data of epidermal thickness evaluated by optical coherence tomography: effects of age, gender, skin type, and anatomic site," J. Dermatol. Sci. 44(3), 145-152 (2006).
32. S. Prahl, "Everything i think you should know about inverse addingdoubling," Oregon Medical Laser Center (2011), http://omlc.org/ software/iad/ (8 February 2016).

33. T. Moffitt, Y. C. Chen, and S. A. Prahl, "Preparation and characterization of polyurethane optical phantoms," J. Biomed. Opt. 11(4), 041103 (2006).

34. A. Fernandez-Oliveras, M. Rubino, and M. M. Perez, "Scattering anisotropy measurements in dental tissues and biomaterials," J. Eur. Opt. Soc. Rapid Publ. 7, 12016 (2012).

35. D. A. Boas and A. K. Dunn, "Laser speckle contrast imaging in biomedical optics," J. Biomed. Opt. 15(1), 011109 (2010).

36. T. Son, J. Lee, and B. Jung, "Contrast enhancement of laser speckle contrast image in deep vasculature by reduction of tissue scattering," J. Opt. Soc. Korea 17(1), 86-90 (2013).

Biographies for the authors are not available. 